A N N A L E S

UNIVERSITATIS MARIAE CURIE-SKŁODOWSKA

LUBLIN - POLONIA

VOL. LXXI SECTIO AAA 2016

\title{
AN EXACT ANALYTICAL SOLUTION TO THE SHALLOW WATER EQUATIONS NEAR BEACHES
}

\author{
M. Yourdkhani ${ }^{1}$ and S. Zarrinkamar ${ }^{2}$ \\ ${ }^{1}$ Civil Engineering Group, Alaodoleh Semnani Institute of Higher Education, Garmsar, Iran \\ ${ }^{2}$ Department of Basic Sciences, Garmsar Branch, Islamic Azad University, Garmsar, Iran \\ m.yourdkhani2003@gmail.com,zarrinkamar.s@gmail.com
}

\begin{abstract}
The nonlinear differential equation governing the dynamics of water waves can be well approximated by a linear counterpart in the case of shallow waters near beaches. The linear equation, which is of second order nature, cannot be exactly solved in many apparently simple cases. In our work, we consider the shape of system as a complete second-order polynomial which contains the constant (step-like), linear and quadratic shapes near the beach. We then apply some novel transformations and transform the problem into a form which can be solved in an exact analytical manner via the powerful Nikiforov-Uvarov technique. The eigenfunctions of the problem are obtained in terms of the Jacobi polynomials and the eigenvalue equation is reported for any arbitrary mode.
\end{abstract}

Keywords: shallow water, wave equation, Nikiforov-Uvarov technique, Jacobi polynomial.

\section{INTRODUCTION}

We frequently face wave equations in various fields of science and engineering including in water and ocean engineering. Although the governing equations in such field are of nonlinear nature, they can be well approximated by linear counterparts in 
some cases. In particular, the shallow-water equations might be described by linear second-order partial differential equations. Shallow-water equations are based on conservation laws and have been so far analyzed by various numerical techniques [1]. When reviewing the existing literature on the subject, we find a variety of numerical techniques have been applied to the field including finite volume [2], Galerkin method [3], Runge-Kutta methods [4], finite element [5], etc [2,6 and many references therein].

The other parallel approach, i.e. the analytical scheme, despite being used, has been less applied to the field. In the analytical framework, we can use the rich ideas of factorization [7], series expansion [8], Lie groups [9], etc. The shallow-water wave equations have also been studied in connection with the tsunami phenomenon [10]. In our work, bearing in mind the significance of shallow-water studies as well as the relative lack of analytical studies in the field, we consider a linear wave equation valid near beaches. For the configuration of the problem, we consider the case of parabolic deformation which is a realistic shape. Next, by applying the common separation technique, we obtain the corresponding linear differential equation. Afterwards, we apply some transformations and bring the problem into a form of a hypergeometric equation. To solve the problem, we use the powerful Nikiforov-Uvarov (NU for short) $[11,12,13]$ technique, which has been widely used for various wave equations of physics, and thereby report the exact analytical eigenfunctions and the corresponding eigenvalue equation.

\section{THE GEOMETRY OF THE SYSTEM AND THE GOVERNING EQUATION}

We study the linear shallow-water equation [14]

$$
\frac{d^{2} \eta}{d t^{2}}+\frac{d}{d x}\left(h(x) \frac{d \eta}{d x}\right)=0
$$

where $\eta(x, t)$ is the wave function and $h(x)$ the depth. A simple separation of variables via gives the second-order ordinary equation

$$
y^{\prime \prime}+\frac{h^{\prime}(x)}{h(x)} y^{\prime}+\frac{\lambda}{h(x)} y=0 .
$$

Considering $h(x)=a x^{2}+b x+c$ in Eq. (2), we have 
$y^{\prime \prime}+\frac{2 a x+b}{a x^{2}+b x+c} y^{\prime}+\frac{\lambda}{a x^{2}+b x+c} y=0$.

Decomposing the second order polynomial in terms of its roots as

$$
a x^{2}+b x+c=a(x-r)(x-s)
$$

where

$r=-\frac{b}{2 a}+\sqrt{\frac{b^{2}}{4 a^{2}}-\frac{c}{a}}$,

$s=-\frac{b}{2 a}-\sqrt{\frac{b^{2}}{4 a^{2}}-\frac{c}{a}}$,

and performing the changing of variable

$$
z=x+\frac{b}{2 a}-\sqrt{\frac{b^{2}}{4 a^{2}}-\frac{c}{a}},
$$

Eq. (3) appears as

$$
y^{\prime \prime}+\frac{1-\frac{1}{\sqrt{\frac{b^{2}}{4 a^{2}}-\frac{c}{a}}} z}{\left(1-\frac{1}{\sqrt{\frac{b^{2}}{a^{2}}-\frac{4 c}{a}}} z\right)} y^{\prime}+\frac{\frac{-\lambda}{\sqrt{b^{2}-4 a c}} z+\frac{\lambda a}{\left(b^{2}-4 a c\right)} z^{2}}{\left(1-\frac{1}{\sqrt{\frac{b^{2}}{a^{2}}-\frac{4 c}{a}}} z\right)^{2}} y=0 .
$$

To solve the rather complicated Eq. (6), we use the NU method introduced in the forthcoming section.

\section{THE NIKIFOROV-UVAROV METHOD}

Within this section, we will introduce the simple but powerful NU technique which has solved many important problems in quantum mechanics $[11,12]$. According to the NU method, a second-order differential equation of the NU method can solve a second-order differential equation of the form [12] 
$\psi_{n}^{\prime \prime}(s)+\frac{\tilde{\tau}(s)}{\sigma(s)} \psi_{n}^{\prime}(s)+\frac{\tilde{\sigma}(s)}{\sigma^{2}(s)} \psi_{n}(s)=0$,

where $\sigma(s)$ and $\tilde{\sigma}(s)$ are polynomials, at most of the second degree, and $\widetilde{\tau}(s)$ is a first-degree polynomial. To make the application of the NU method simpler and more direct, we introduce a more compact presentation of the idea. In order to do this, we rewrite Eq. (1) as follows [11]

$\psi_{n}^{\prime \prime}(s)+\left(\frac{c_{1}-c_{2} s}{s\left(1-c_{3} s\right)}\right) \psi_{n}^{\prime}(s)+\left(\frac{-\xi_{1} s^{2}+\xi_{2} s-\xi_{3}}{s^{2}\left(1-c_{3} s\right)^{2}}\right) \psi_{n}(s)=0$,

in which

$\psi_{n}(s)=\phi(s) y_{n}(s)$

Comparing Eq. (1) with Eq. (2), we obtain the following identifications:

$$
\begin{aligned}
& \tilde{\tau}(s)=c_{1}-c_{2} s, \\
& \sigma(s)=s\left(1-c_{3} s\right), \\
& \tilde{\sigma}(s)=-\xi_{1} s^{2}+\xi_{2} s-\xi_{3},
\end{aligned}
$$

Following the NU method $[12,13]$, we obtain the following required parameters:

(i) the relevant constant:

$$
\begin{aligned}
& c_{4}=\frac{1}{2}\left(1-c_{1}\right), \\
& c_{6}=c_{5}^{2}+\xi_{1}, \\
& c_{8}=c_{4}^{2}+\xi_{3}, \\
& c_{10}=c_{1}+2 c_{4}+2 \sqrt{c_{8}} \\
& c_{12}=c_{4}+\sqrt{c_{8}} \\
& c_{13}=c_{5}-\left(\sqrt{c_{9}}+c_{3} \sqrt{c_{8}}\right),
\end{aligned}
$$$$
c_{5}=\frac{1}{2}\left(c_{2}-2 c_{3}\right) \text {, }
$$$$
c_{7}=2 c_{4} c_{5}-\xi_{2} \text {, }
$$$$
c_{9}=c_{3} c_{7}+c_{3}^{2} c_{8}+c_{6} \text {. }
$$$$
c_{11}=c_{2}-2 c_{5}+2\left(\sqrt{c_{9}}+c_{3} \sqrt{c_{8}}\right)
$$

(ii) the essential polynomial functions: 


$$
\begin{aligned}
& \pi(s)=c_{4}+c_{5} s-\left[\left(\sqrt{c_{9}}+c_{3} \sqrt{c_{8}}\right) s-\sqrt{c_{8}}\right], k=-\left(c_{7}+2 c_{3} c_{8}\right)-2 \sqrt{c_{8} c_{9}}, \\
& \tau(s)=c_{1}+2 c_{4}-\left(c_{2}-2 c_{5}\right) s-2\left[\left(\sqrt{c_{9}}+c_{3} \sqrt{c_{8}}\right) s-\sqrt{c_{8}}\right], \\
& \tau^{\prime}(s)=-2 c_{3}-2\left(\sqrt{c_{9}}+c_{3} \sqrt{c_{8}}\right)<0 .
\end{aligned}
$$

(iii) The energy equation:

$$
\begin{aligned}
& c_{2} n-(2 n+1) c_{5}+(2 n+1)\left(\sqrt{c_{9}}+c_{3} \sqrt{c_{8}}\right)+ \\
& n(n-1) c_{3}+c_{7}+2 c_{3} c_{8}+2 \sqrt{c_{8} c_{9}}=0
\end{aligned}
$$

(iv) The wave functions

$$
\begin{aligned}
& \rho(s)=s^{c_{10}}\left(1-c_{3} s\right)^{c_{11}}, \phi(s)=s^{c_{12}}\left(1-c_{3} s\right)^{c_{13}}, c_{12}>0, c_{13}>0, \\
& y_{n}(s)=P_{n}^{\left(c_{10}, c_{11}\right)}\left(1-2 c_{3} s\right), c_{10}>-1, c_{11}>-1, \\
& \psi_{n \kappa}(s)=N_{n \kappa} s^{c_{12}}\left(1-c_{3} s\right)^{-c_{12}-\frac{c_{13}}{c_{3}}} P_{n}^{\left(c_{10}-1, \frac{c_{11}}{c_{3}}-c_{10}-1\right)}\left(1-2 c_{3} s\right),
\end{aligned}
$$

where $P_{n}^{(\mu, v)}(x), \mu>-1, v>-1$, and $x \in[-1,1]$ are Jacobi polynomials with

$$
P_{n}^{(\alpha, \beta)}(1-2 s)=\frac{(\alpha+1)_{n}}{n !}{ }_{2} F_{1}(-n, 1+\alpha+\beta+n ; \alpha+1 ; s)
$$

where

$$
P_{n}^{(\alpha, \beta)}(x)=\frac{\Gamma(\alpha+n+1)}{n ! \Gamma(\alpha+\beta+n+1)} \sum_{m=0}^{n}\left(\begin{array}{l}
n \\
m
\end{array}\right) \frac{\Gamma(\alpha+\beta+n+m+1)}{\Gamma(\alpha+m+1)}\left(\frac{x-1}{2}\right)^{m}
$$

and $N_{n \kappa}$ is a normalization constant. Also, the above wave functions can be expressed in terms of the hypergeometric function via

$$
\psi_{n \kappa}(s)=N_{n \kappa} s^{c_{12}}\left(1-c_{3} s\right)^{c_{13}}{ }_{2} F_{1}\left(-n, 1+c_{10}+c_{11}+n ; c_{10}+1 ; c_{3} s\right)
$$

where $c_{12}>0, c_{13}>0$ and $s \in\left[0,1 / c_{3}\right], c_{3} \neq 0$. 


\section{THE EXACT ANALYTICAL SOLUTIONS}

A simple comparison with Eq. indicates the correspondence

$$
\begin{aligned}
& c_{1}=1, c_{2}=\frac{1}{\sqrt{\frac{b^{2}}{4 a^{2}}-\frac{c}{a}}}, c_{3}=\frac{1}{\sqrt{\frac{b^{2}}{a^{2}}-\frac{4 c}{a}}}, \\
& \xi_{3}=0, \xi_{2}=\frac{\lambda}{\sqrt{b^{2}-4 a c}}, \xi_{1}=\frac{-\lambda a}{\left(b^{2}-4 a c\right)} .
\end{aligned}
$$

Therefore, the rest of required parameters are obtained as

$$
\begin{aligned}
& c_{4}=0, \quad c_{5}=0, \quad c_{6}=\frac{-\lambda a}{\left(b^{2}-4 a c\right)}, \quad c_{7}=-\frac{\lambda}{\sqrt{b^{2}-4 a c}}, \\
& c_{8}=0, \quad c_{9}=-\frac{2 \lambda a}{b^{2}-4 a c}, c_{10}=1 \\
& c_{11}=\frac{1}{\sqrt{\frac{b^{2}}{4 a^{2}}-\frac{c}{a}}}+2 \sqrt{\frac{-2 \lambda a}{\left(b^{2}-4 a c\right)}}, \\
& c_{12}=0, c_{13}=-\sqrt{-\frac{2 \lambda a}{\left(b^{2}-4 a c\right)}} .
\end{aligned}
$$

Therefore, the eigenfunctions are

$$
\psi_{n \kappa}(s)=N_{n \kappa}\left(1-\frac{a}{\sqrt{b^{2}-4 a c}} s\right)^{\sqrt{\frac{2 \lambda}{(a)}}} P_{n}^{\left(0, \sqrt{\frac{-8 \lambda}{a\left(b^{2}-4 a c\right)}}\right)}\left(1-\frac{2 a}{\sqrt{b^{2}-4 a c}} s\right),
$$

The eigenvalue problem can be directly found as

$$
\frac{1}{\sqrt{\frac{b^{2}}{4 a^{2}}-\frac{c}{a}}} n+(2 n+1)\left(\sqrt{-\frac{2 \lambda a}{b^{2}-4 a c}}\right)+\frac{1}{\sqrt{\frac{b^{2}}{a^{2}}-\frac{4 c}{a}}} n(n-1)-\frac{\lambda}{\sqrt{b^{2}-4 a c}}=0 .
$$




\section{CONCLUSIONS}

We considered a linear differential equation governing the motion of shallow waters near beaches. For the configuration of the problem, we assumed a parabolic shape and via some transformations, transformed the problem into a hypergeometric-type equation. Next, using the Nikiforov-Uvarov technique, the eigenfunctions were reported in terms of the Jacobi polynomials and the corresponding eigenvalue equation was reported.

\section{REFERENCES}

1. Gallouet Th. J., Herard M. and Seguin N., Computers \& Fluids 32, 479 (2003).

2. Garcia-Navarro P. and Vazquez-Cendon M. E. Computers \& Fluids 29, 951(2000).

3. Aizinger V. and Dawson C., Comp. Meth. App. Mech. Eng. 196, 734 (2007).

4. Butcher J. C., App. Num. Math. 20, 247 (1996).

5. Gourgue O. et al., Adv. Water Res. 32, 1726 (2009).

6. Vreugdenhil C. B., Numerical methods for shallow-water waves, volume 13 of Water Science and Technology Library, Kluwer Academic Publishers (1994).

7. Shi-Hai Dong, Factorization Methods in Quantum Mechanics, Springer-Verlag, New York (2007).

8. Shi-Hai Dong, Wave Equations in Higher Dimensions, Springer-Verlag, New York (2011).

9. Olver P. J. Applications of Lie Groups to Differential Equations, Springer-Verlag, New York (1986).

10. Liu P. L.-F., Lynett P. and Synolakis C. E., J. Fluid Mech., vol. 478, pp. 101-109 (2003).

11. Nikiforov A. F. and Uvarov V. B., Special Functions of Mathematical Physics Birkhauser, Basel (1988).

12. Tezcan C. and Sever R., Int. J. Theor. Phys. 48, 337 (2009).

13. Antia A. D., Akpan I. O., Ikot A. N., Maghsoodi E., Zarrinkamar S., Hassanabadi H., Annales Universitatis Mariae Curie-Skłodowska, sectio AAA-Physica 70, 167 (2015).

14. Mei Ch. C., The Applied Dynamics of Ocean Surface Waves, John Wiley \& Sons, New York (1983). 